\title{
Lignin Valorization: Concentration of Model Phenolic Compounds by Nanofiltration
}

\author{
Elson Dinis Gomes, P. C. R. Pinto, Alírio E. Rodrigues \\ Department of Chemical Engineering, Faculty of Engineering University of Porto, Porto, \\ Portugal (elson@fe.up.pt)
}

\begin{abstract}
Lignin is a biopolymer found in plants which can be valorized as a source of value added compounds by breaking its structure in the constituting monomers. A model solution was prepared with seven phenolic compounds, including vanillin and syringaldehyde, usually found after alkaline lignin oxidation. The model solution was concentrated by nanofiltration membranes with low molecular weight cut-off (MWCO). Two membranes (DOW NF270 and KOCH MPS-34) were tested for permeate fluxes and phenolic compound rejection. Significant reduction of the volume mixture was achieved with high permeate fluxes as well as high rejections (above 90\%) for the phenolic compounds studied. The membrane with the best performance was the DOW NF270 with maintained high rejections while having higher permeate fluxes resulting in higher productivity when compared with the other membrane KOCH MPS-34 also tested.
\end{abstract}

Subject Headings. Chemical Industry, Biotechnology, Polymer

Author Keywords. Lignin, Valorization, Value Added Compounds, Vanillin, Syringaldehyde, Nanofiltration

\section{Introduction}

Lignin is one of the main constituents of wood second only to cellulose. The exact composition varies with the plant origin but eucalyptus can have up to $25 \%$ mass, dry basis, of wood as lignin. Lignin composition depends mostly on the biomass origin (Watkins, 2015). Other biomass other than softwood (e.g.: pines) and hardwoods (e.g: eucalyptus) like annual plants (e.g: grass, tobacco stalks) can be used as sources of lignin. Lignin is a randomly cross-linked polymer of phenylpropane units. It is composed by the monomer precursors $p$-coumaryl, coniferyl and sinapyl alcohols presented in Figure 1 (Doherty, 2011). Lignin is produced in abundance in the pulp and paper industry since it is a side stream in the main process of obtaining the cellulose. The main process employed in the pulp and paper industry is the Kraft process where the process chemicals are recycled and cellulose is washed out and the lignin remains solubilized in the black liquor due to high alkalinity of the medium. The black liquor is then concentrated in a multistage evaporation sequence and burned for energy valorization due to lignin high heating value, usually (Telmo, 2011). The burning of the concentrated black liquor constitutes a bottleneck in the production of cellulose in the industry since the furnaces have a limited capacity to burn liquor and to produce vapor, so the decrease of the calorific content of the liquor is desired. This could be achieved if some of this lignin is also precipitated by acidification. There are two main processes for removing lignin from the liquor the LignoBoost and the LignoForce (Nagy, 2010) both employing acidification at some point. 
Usually the pulp and paper industry has a surplus of electric energy due to the high quantities of black liquor that needs to be burned (Orr,1995).

Lignin being a polymer, can be deconstructed to give off value added chemicals like vanillin, syringaldehyde and apocynin. These monomer units are themselves precursors for other materials including active principles for drugs, for instance syringaldehyde can be used to produce trimethoprim, an antifungal drug and vanillin can be used simply as flavoring agent or can also be employed in the production of pharmaceuticals like L-dopa and ALDOMET ${ }^{\circledR}$ (Hocking,1997). There are several ways to deconstruct the polymer but each approach has its drawbacks as well. The most common way is subjecting the liquor or lignin to both high temperature and pressure along with a gas oxidant being bubbled, typically pure oxygen or air (Fargues,1996).
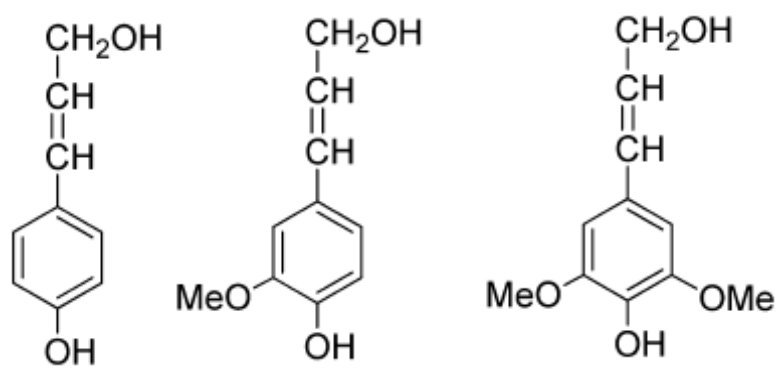

Figure 1: Building blocks of lignin: p-coumaryl, coniferyl and sinapyl alcohols

Since the first publications on the subject of obtaining value added compounds from lignin (Mathis,1995), the concept of biorefinery has been explored by several other authors. The highly integration of several processes would be necessary, much like what happens nowadays with the chemical industry, to valorize all of the biological sources of carbon like cellulose, hemicellulose and lignin (Doherty,2011). Thus the pulp and paper industry has the potential to supply the chemical industry with natural derived building blocks (monomer and polymer) originating in the undervalued lignin.

Nanofiltration can potentially give a contribution to the biorefinery concept by concentrating the oxidized solutions and allowing the treatment of smaller volumes downstream. Provided that the molecular weight cut-off of the membrane is in the range of the dimension of the molecules in the solution, rejection of these is expected. Filtration has been used to concentrate several other low molecular weight products like ellagic acid (Benítez,2005) but to our knowledge no attempt to concentrate phenolic compounds derived from lignin has been performed.

In this work the performance of two membranes (KOCH MPS-34 and DOW NF270) was studied in order to select the best membrane for the concentration of the phenolic compounds usually found in Kraft black liquors. In order to do that, the rejections and the permeate fluxes were determined in each nanofiltration experiment for both membranes.

\section{Materials and Methods}

\subsection{Model solution}

The initial solutions to be processed in the membranes were prepared with the phenolic compounds derived from lignin: $p$-hydroxybenzaldehyde $(H)$, vanillic acid (VA), vanillin (V), syringic acid (SA), syringaldehyde (S), acetovanillone (VO) and acetosyringone (SO). A total of 11 liters of solution was prepared for each experiment. The concentration of each phenolic compound is presented in Table 1 . Also the mixture's pH was adjusted to 10 with $1 \mathrm{M} \mathrm{NaOH}$ 
solution to mimic the alkaline media that the membrane would be subjected to if the real oxidation mixture were to be processed.

\begin{tabular}{lc}
\hline \multicolumn{1}{c}{ Phenolic compound } & Concentration, g/L \\
\hline vanillin (V) & 0.500 \\
\hline syringaldehyde (S) & 0.500 \\
\hline vanillic acid (VA) & 0.100 \\
\hline syringic acid (SA) & 0.100 \\
\hline p-hydroxybenzaldehyde (H) & 0.050 \\
\hline acetovanillone (VO) & 0.050 \\
\hline acetosyringone (SO) & 0.050 \\
\hline
\end{tabular}

Table 1: Concentration of the seven phenolic compounds in the synthetic mixture

\subsection{Installation}

The nanofiltrations were carried out in a SEPA CF II, GE Osmonics module, USA. The module is composed by a diaphragm pump, a flowmeter, a hydraulic hand pump, and a hydraulic press that holds the membrane support. The equipment withstands a maximum operating pressure of $69 \mathrm{bar}$ and a maximum operating temperature of $170^{\circ} \mathrm{C}$. The effective membrane area available is $144 \mathrm{~cm}^{2}$. The nanofiltrations were performed in recirculation mode where the retentate is returned to the feed tank and keeps recirculating.

\subsection{Membranes}

The membranes used were both nanofiltration membranes which have a molecular weight cut off (MWCO) just above the molecular weights of the phenolic compounds to be concentrated. The remaining characteristics of the membranes are given in Table 2 . Since the phenolic compounds to be concentrated have molecular weights between $122.12 \mathrm{~g} / \mathrm{mol}$ and $198.17 \mathrm{~g} / \mathrm{mol}$, the membranes selected were supposed to have a MWCO on the range of the molecules to be concentrated by nanofiltration. Although the membranes have different rated MWCO it should be pointed that MWCO is not the best indications as to whether a molecule will permeate the membrane since other effects are also present. All membranes are received as flat sheets and are cut to the dimensions of the module.

\begin{tabular}{lcc}
\multicolumn{1}{c}{ Characteristics } & DOW NF270 & KOCH MPS-34 \\
\hline MWCO & 400 & 200 \\
\hline Material & Polyamide & Not available \\
\hline pH range & 2 to 11 & 0 to 14 \\
\hline Maximum Pressure & 40 & 35 \\
\hline Maximum Temperature & $50^{\circ} \mathrm{C}$ & $70^{\circ} \mathrm{C}$ \\
\hline \multicolumn{2}{c}{ Table 2: Membrane characteristics for DOW NF270 } \\
and KOCH MPS-34 nanofiltration membranes
\end{tabular}

\subsection{Analysis}

Samples obtained along the experiments were extracted by solid phase extraction (SPE) and analyzed by high-performance liquid chromatography (HPLC). The HPLC is equipped with a diode array detector and the detection wavelength is set at $280 \mathrm{~nm}$. The samples were prepared following a procedure detailed previously by Pinto et al (Pinto, 2010).

\subsection{Operation}

All membranes are placed in water overnight, previous to membrane installation. Then the membrane was placed on the support and a transmembrane pressure of 40 bar was applied 
on the hydraulic press to seal the membrane support. Water was circulated while the pressure in the module was increased in steps of 3 bar/10 min until 30 bar and left at 32 bar for 90 min. Then $1 \mathrm{~L}$ of the prepared solution is recirculated on the system for $10 \mathrm{~min}$ before the $10 \mathrm{~L}$ are treated. The experiments were carried at 30 bar and temperature ranged from $22^{\circ} \mathrm{C}$ to $28^{\circ} \mathrm{C}$. Samples of the permeate and retentate were collected at the same time for each point. The nanofiltration was stopped after $10 \mathrm{~L}$ of permeate was recovered.

\section{Results and Discussion}

The first membrane tested, KOCH MPS-34 allowed the concentration of the initial model solution, concluding the nanofiltration experiment with only $0.980 \mathrm{~L}$ of the initial $10 \mathrm{~L}$ of model solution. The evolution of the nanofiltration regarding the increase in concentration of the seven phenolic compounds studied is presented in Figure 2 . A volume reduction factor of 10 was achieved. The phenolic compounds with more expression are vanillin and syringaldehyde as these were the compounds in higher concentration in the initial solution. The increase in concentration is also in agreement with the other compounds in lower concentrations like the ketones (VO and SO), the acids (VA and SA) and the aldehyde in lower concentration, $\mathrm{H}$.

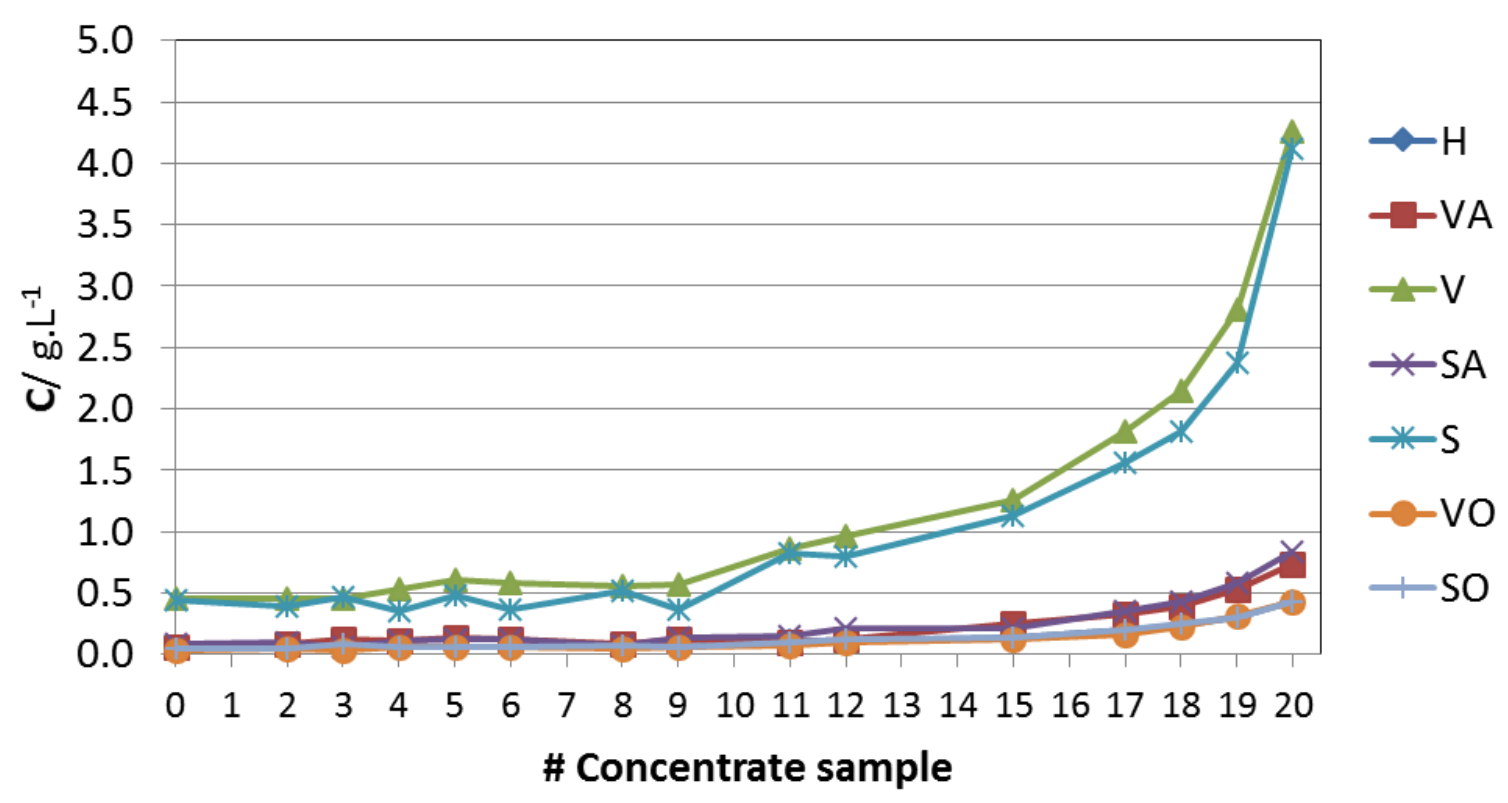

Figure 2: Concentration of the seven phenolic compounds followed in the retentate along the experiment with the $\mathrm{KOCH}$ MPS-34. Conditions: temperature from $26.9^{\circ} \mathrm{C}$ to $28.0^{\circ} \mathrm{C}$, pressure of $30 \mathrm{bar}$, flowrate on retentate side $=252 \mathrm{~L} \mathrm{~min}-1$, average permeate flux $=60 \mathrm{~L} \mathrm{~m}-2 \mathrm{~h}-1$. Total experiment time: $10 \mathrm{~h}$

The experiment with the second membrane, DOW NF270, is also presented. The evolution of this experiment at 20 bar is given in Figure 3. The same behavior is observed ending with similar values of concentrated solution to the solution concentrated with the KOCH MPS-34 nanofiltration membrane. In this experiment also a volume reduction factor of 10 was achieved. 


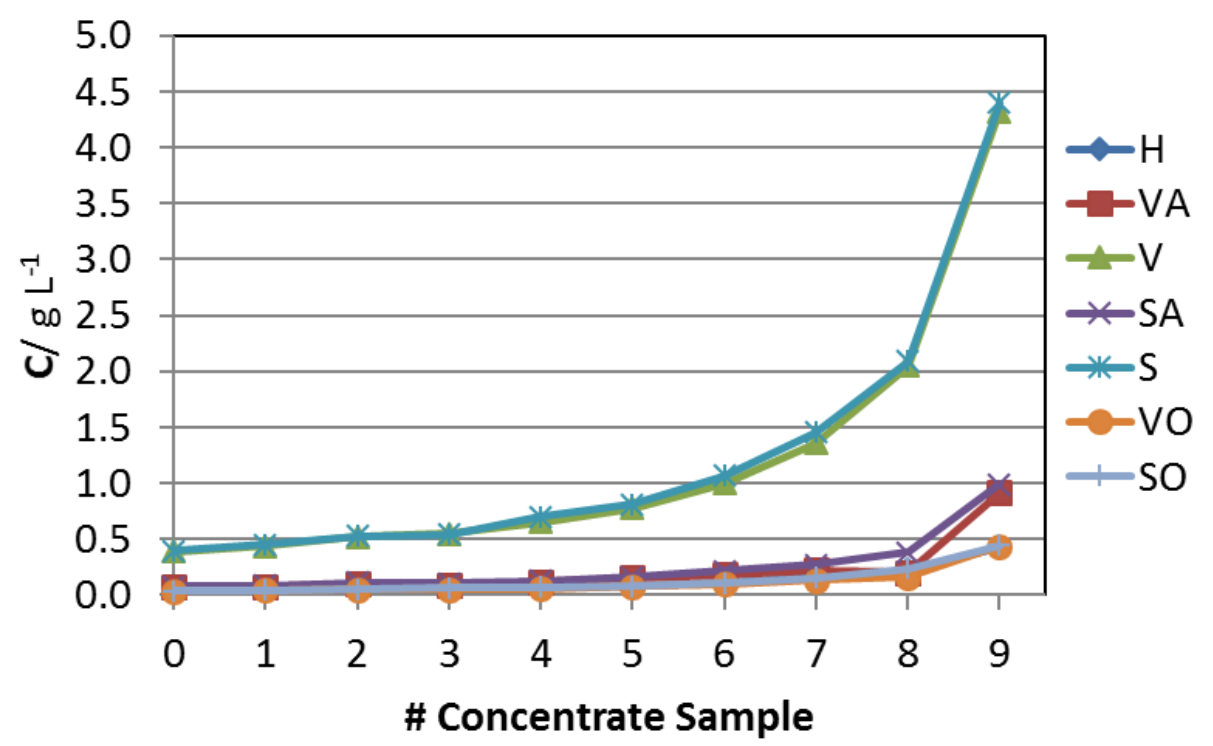

Figure 3: Concentration of the seven phenolic compounds followed in the retentate along the experiment with the DOW NF270. Conditions: temperature from $25.7^{\circ} \mathrm{C}$ to $28.6^{\circ} \mathrm{C}$, pressure of 20 bar, flowrate on retentate side $=252 \mathrm{~L}$ min-1, average permeate flux $=280 \mathrm{~L} \mathrm{~m}-2 \mathrm{~h}-1$. Total experiment time: $2.4 \mathrm{~h}$

The performance of the membranes was assessed through rejection. The rejection of the membranes is calculated according to equation (1). Where $C_{p}$ is the concentration of permeate and $C_{r}$ is the concentration on the retentate (or concentrate). It is a measure of the capacity to retain, in this case, the phenolic compounds in study.

$$
\text { Rejection }(\%)=100 \times\left(1-\frac{C_{p}}{C_{r}}\right)
$$

In Figure 4, the rejections obtained for the two membranes studied in the four experiments are presented. All of the experiments resulted in high rejections for all of the phenolic compounds studied. $p$-hydroxybenzaldehyde is the compound with the lowest rejection for both membranes in all experiences due to its lower molecular weight and lower stereoblocking due to the lacking of a methoxy group that is present in all of the other six compounds present, twice in compounds derived from sinapyl alcohols or syringyl units like $\mathrm{SA}, \mathrm{S}$ and SO.

Rejections for the membrane with the lower MWCO, KOCH MPS-34, are higher than those for the DOW NF270. Also worth noting is that in the experiments at lower transmembrane pressures, 20 bar, the rejections increase, overall. This increase due to lower transmembrane pressure is more evident for $p$-hydroxybenzaldehyde. 


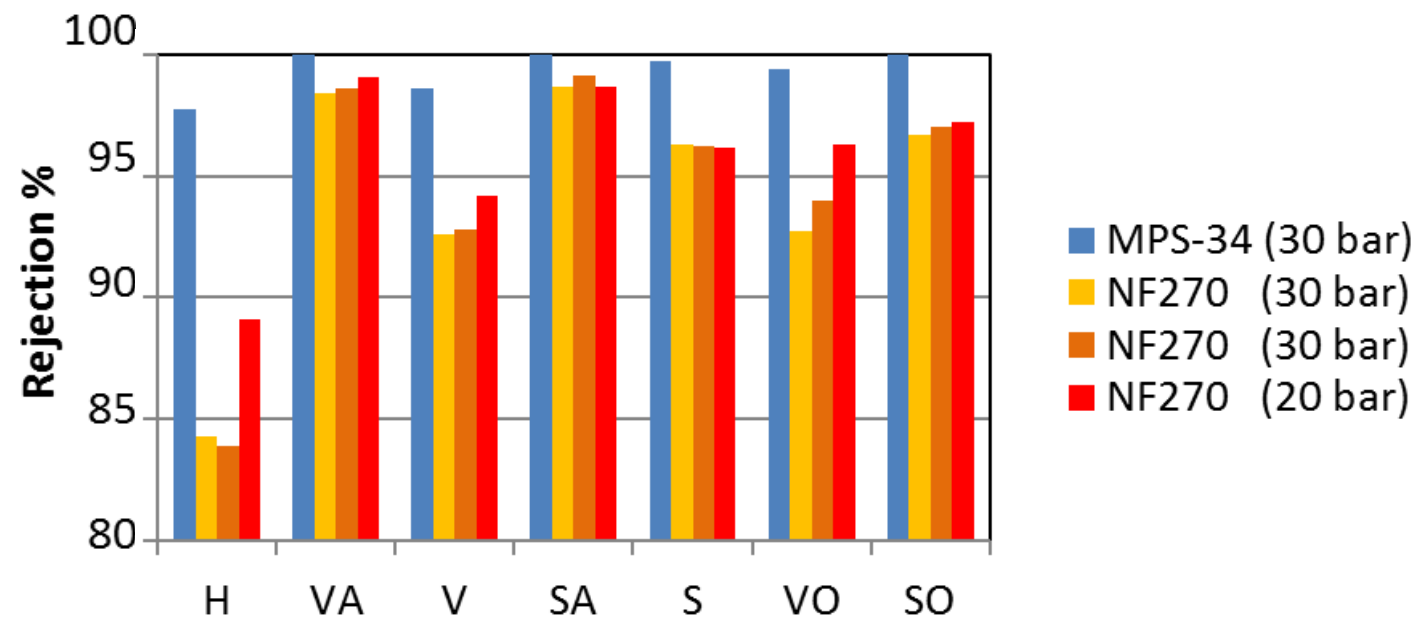

Figure 4: Comparison of the membranes rejections towards each compound in the four experiments (membranes KOCH MPS-34 and DOW NF270). Last permeate and retentate used for determination of rejection

In Figure 5 the permeate fluxes of both membranes are compared (also at 20 bar for the DOW NF270). The performance of the several membranes is compared regarding the time each membrane took to complete the concentration of the solution over 10 times. It is clear that even at a lower transmembrane pressure of 20 bar the DOW NF270 performs better than the $\mathrm{KOCH}$ MPS-34 at 30 bar. The DOW NF270 allows the processing of the model solution in just $2.40 \mathrm{~h}$ operating at 20 bar while the KOCH MPS-34 experiment at 30 bar finishes after $10.00 \mathrm{~h}$. When the DOW NF270 operates at 30 bar this difference is even greater finishing the experiment after just $1.75 \mathrm{~h}$ of operation.

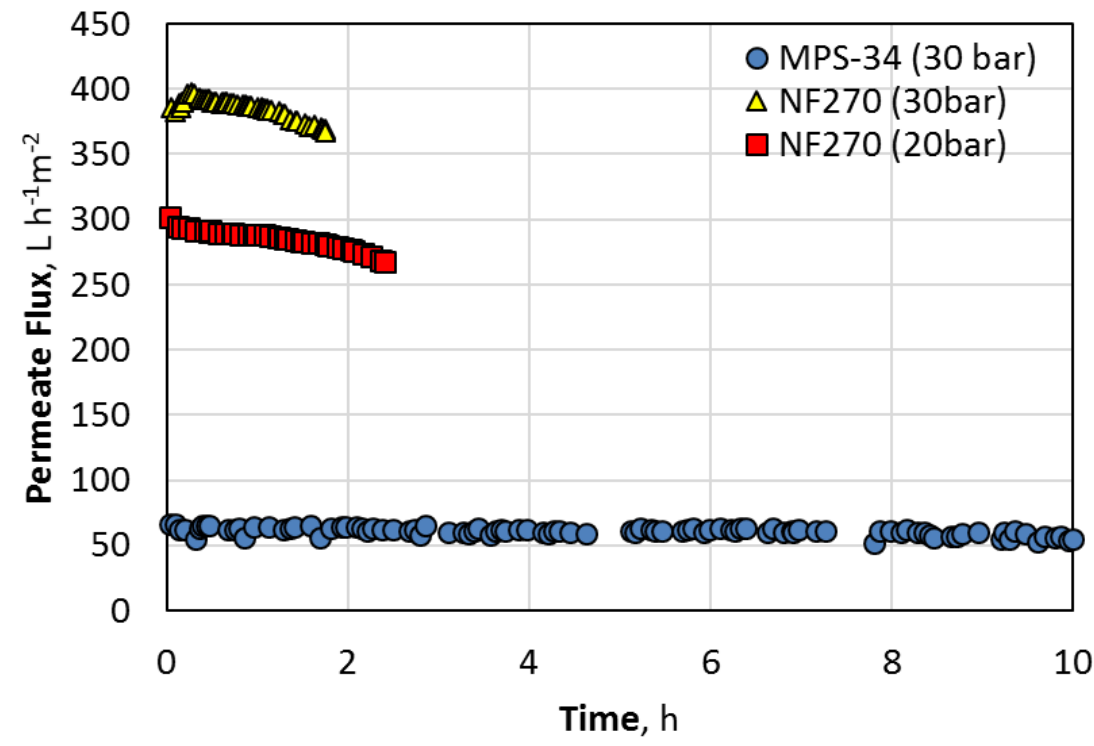

Figure 5: Comparison of the permeate fluxes of the different membranes

\section{Conclusions}

A model solution was prepared with seven model compounds usually found in Kraft black liquors after undergoing oxidation. The solution was successfully concentrated over 10 times the initial concentrations. Higher permeate fluxes were obtained with the DOW NF270 nanofiltration membrane when compared with the KOCH MPS-34. Also the rejections remained high for the membrane with the higher permeate flux (DOW NF270) with rejection values above $92 \%$ for six of the seven compounds (VA, V, SA, S, VO, SO) while for $H$ the rejection was $84.3 \%$. 
The ability of these membranes to concentrate this solution open up the possibility to work with significantly smaller volumes of mixture when processing real mixtures but real mixtures may also be more prone to fouling since oligomer structures are present as well.

\section{References}

Araújo, J. D. (2008) Production of vanillin from lignin present in the Kraft black liquor of the pulp and paper industry, PhD Thesis in Biological and Chemical Engineering, Faculty of Engineering, University of Porto, Portugal.

Benítez,F. J., Acero, J. L., Leal, A. I., Real, F. J., (2005) Purification of Ellagic Acid by UF Membranes. Chem. Eng. Technol., 28, 1035-1040.

Doherty, W.O.S., Mousavioun, P., Fellows, C.M., (2011) Value-adding to cellulosic ethanol: lignin polymers. Ind. Crops Prod., 33 (2) pp. 259-276

Fargues, C., Mathias. Á., Rodrigues, A., (1996) Kinetics of Vanillin Production from Kraft Lignin Oxidation. Industrial \& Engineering Chemistry Research 35 (1), 28-36 DOI: 10.1021/ie950267k.

Hocking, Martin B., (1997) Vanillin: Synthetic Flavoring from Spent Sulfite Liquor. Journal of Chemical Education 74 (9), 1055, DOI: 10.1021/ed074p1055.

Nagy, M., Kosa, M., Theliander, H., and Ragauskas, A. J., (2010) Characterization of CO2 precipitated Kraft lignin to promote its utilization. Green Chem., 12, 31.

Mathias, A. L., Rodrigues, A. E., (1995) Production of Vanillin by Oxidation of Pine Kraft Lignins with Oxygen. Holzforschung - International Journal of the Biology, Chemistry, Physics and Technology of Wood. Volume 49, Issue 3, Pages 273-278, ISSN (Online) 1437-434X, ISSN (Print) 0018-3830, DOI: 10.1515/hfsg.1995.49.3.273.

Orr, A., Simons, H. A., (1995) Energy Generation and Use in the Kraft Pulp Industry, H. A SIMONS LTD, ACEEE Proceedings, pp. 191-203.

Pinto, P. C. R., da Silva, E. A. B., Rodrigues, A. E. (2010) Comparative study of solid-phase extraction and liquid-liquid extraction for the reliable quantification of high value added compounds from oxidative processes of wood-derived lignin. Ind. Eng. Chem. Res 49(23): 12311-12318.

Telmo, C., Lousada, J., (2011) The explained variation by lignin and extractive contents on higher heating value of wood. Biomass Bioenergy, 35(5), 16632011.

Watkins, D., Nuruddin, M., Hosur, M., Tcherbi-Narteh, A., Jeelani, S. (2015) Extraction and characterization of lignin from different biomass resources. J. Mater. Res. Technol., 4, pp. 26-32. DOI: 10.1016/j.jmrt.2014.10.009. 